\title{
Usefulness of plasma D-dimer level for monitoring development of distant organ metastasis in colorectal cancer patients after curative resection
}

This article was published in the following Dove Press journal:

Cancer Management and Research

\section{Yi Guo \\ Feng Chen \\ Wei Cui}

State Key Laboratory of Molecular Oncology, Department of Clinical Laboratory, National Cancer Center/ National Clinical Research Center for Cancer/Cancer Hospital, Chinese Academy of Medical Sciences and Peking Union Medical College, Beijing, I0002I, China

Video abstract

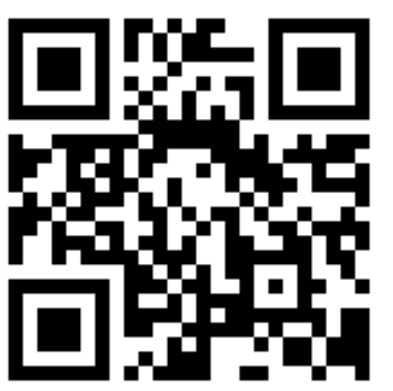

Point your SmartPhone at the code above. If you have a QR code reader the video abstract will appear. Or use: http://youtu.be/asI rKjJkQfe

Correspondence: Wei Cui State Key Laboratory of Molecular Oncology, Department of Clinical Laboratory, National Cancer Center/ National Clinical Research Center for Cancer/Cancer Hospital, Chinese Academy of Medical Sciences and Peking Union Medical College, No. I7, Panjiayuan Nanli, Chaoyang District, Beijing, I0002I, China

Tel +8601087788448

Email wendycuiwei@sina.cn
Purpose: To investigate the usefulness of plasma D-dimer level for monitoring the development of distant organ metastasis in colorectal cancer (CRC) patients after curative resection.

Patients and methods: One hundred and seventy-eight CRC patients after curative resection were enrolled in the study. Ninety-two patients developed distant organ metastasis during follow-up (metachronous metastasis), and blood was collected on the day metastasis was confirmed. Eightysix patients had no evidence of metastasis yet, and their blood samples were evaluated at last return visit. The levels of D-dimer, carcinoembryonic antigen (CEA), and lactate dehydrogenase (LDH) between two patient groups were compared. The agreement between D-dimer and CEA (or LDH) was examined. The receiver operator characteristic (ROC) curve was used to evaluate the performance of D-dimer, CEA, LDH, and their combination in detection of distant organ metastasis.

Results: The level of D-dimer in CRC patients with metachronous metastasis was higher than that in non-metastasis patients $(P<0.0001)$. Agreement between D-dimer and CEA was fair $(\kappa=0.416, P<0.0001)$. D-dimer had a larger area under ROC (AUC) $(0.85)$ compared to CEA (0.72) or LDH (0.68). The specificity of D-dimer (73.3\%) was lower than that of CEA $(74.4 \%)$, but the sensitivity (88.0\%) of D-dimer assay was superior to that of CEA assay (65.2\%). LDH showed the lowest sensitivity (42.4\%) and highest specificity (95.3\%) among the three biomarkers. The sensitivity and negative predictive value (NPV) of a combination assay (either D-dimer elevation or CEA elevation) were $94.6 \%$ and $91.1 \%$, respectively, and the specificity and positive predictive value of another combination assay (both D-dimer elevation and LDH elevation) were $97.7 \%$ and $94.9 \%$, respectively. Parallel test of the three markers improved the sensitivity and NPV to $95.7 \%$ and $92.7 \%$, respectively.

Conclusion: Combining with CEA and/or LDH, D-dimer could be a useful surveillance marker for distant organ metastasis in CRC patients after curative resection.

Keywords: D-dimer, colorectal cancer, curative resection, distant organ metastasis, CEA

\section{Introduction}

Colorectal cancer (CRC) is the third most common cancer worldwide, and its mortality rate continues to increase yearly in less developed regions. ${ }^{1}$ According to a report concerning $\mathrm{CRC}$ mortality in China, ${ }^{2}$ the adjusted death rate increased gradually after 2009 , with a mortality of 6.15 per 100,000 people in 2009 which further increased to 7.77 per 100,000 in 2011. Distant organ metastasis from CRC is mainly responsible for the high rate of death. ${ }^{3}$ Approximately $40 \%-50 \%$ of CRC patients will eventually develop distant organ metastasis after resection of the primary tumor (metachronous metastasis). ${ }^{3,4}$ Therefore, it is necessary for these CRC patients to monitor distant organ metastasis during postoperative visit. 
Currently, pathological evidence of malignant cells in distant organs is regarded as the gold standard in CRC metachronous metastasis diagnosis. Imaging techniques, like CT and ultrasound, are commonly used methods for diagnosing distant organ metastasis. ${ }^{5}$ By comparison, blood tests are relatively non-invasive, and patients do not suffer from ionizing radiation and high cost. ${ }^{6}$ Therefore, using serum/plasma biomarkers for detection is more suitable for long-term follow-up. Carcinoembryonic antigen (CEA) is the routine clinical biomarker for predicting metachronous metastasis in CRC, and elevated lactate dehydrogenase $(\mathrm{LDH})$ is also reported as a risk factor for development of distant organ metastasis in patients with $\mathrm{CRC} . .^{7-9}$ According to different cutoff values, the sensitivity of CEA or LDH used as postoperative surveillance ranged from $51.7 \%$ to $70.4 \% .^{9-11}$ Thus, more valuable biomarkers are needed to monitor the development of distant organ metastasis in CRC patients after curative resection.

It is widely accepted that certain CRC patients present coagulation and fibrinolysis abnormalities. ${ }^{12} \mathrm{D}$-dimer can reflect activation of coagulation and fibrinolysis system, as it is a degradation product of cross-linked fibrin. Recently, plasma D-dimer level could be determined routinely in clinical laboratories on the basis of several detection kits. Thus, more and more studies find that higher D-dimer levels before operations are correlated with a more advanced CRC tumor stage $^{10,13-18}$ and a poorer prognosis. ${ }^{14,16,19-23}$ This is more distinct in the cases of distant organ metastasis, which may result from the increased angiogenesis in the primary tumor as well as the formation of tumor emboli in the circulation. ${ }^{18}$ Therefore, we speculate that D-dimer levels might be elevated in CRC patients who develop distant organ metastasis. However, the usefulness of D-dimer for monitoring the development of distant organ metastasis in CRC patients after curative resection has not been reported yet.

In the present study, we measured the plasma D-dimer levels of CRC patients with or without metachronous metastasis after operation. The aim was to evaluate the usefulness of D-dimer combined with CEA and/or LDH as a surveillance marker in detection of distant organ metastasis in CRC after curative resection.

\section{Patients and methods}

\section{Ethics statement}

Oral and written informed consent was provided by all enrolled patients. This study methodology was approved by the Institution Ethics Review Board for Human Studies at the
National Cancer Center/Cancer Hospital, Chinese Academy of Medical Sciences, and Peking Union Medical College. We also have complied with the World Medical Association Declaration of Helsinki regarding ethical conduct of research involving human subjects.

\section{Patients}

The medical information of patients diagnosed with CRC in the Cancer Hospital Chinese Academy of Medical Sciences was reviewed from January 2015 to August 2017. The following inclusion criteria were applied: 1) patients who had undergone primary tumor resection with systematic lymph node dissection for a curative intent, 2) who were histologically confirmed by endoscopic or surgical specimen, and 3) who had complete follow-up data. Cases with following conditions were excluded: 1) patients with concomitant malignant diseases, 2) with infections or other inflammatory diseases, 3) with coagulation dysfunction, 4) being treated with anticoagulants or thrombolytic medications, and 5) with distant organ metastasis initially (synchronous metastasis). Distant organ metastasis was defined as metastatic lesion(s) in organs beyond the colorectum. The identification of metastatic lesion(s) was based on the results of imaging examination, image-guided biopsy, or exploratory laparotomy. Finally, the enrolled cohort comprised 178 patients.

\section{Blood collection and biochemical assays}

Blood samples from peripheral vein were collected at each return visit after surgical operation, and the values of D-dimer, CEA, and LDH were measured. For patients with distant organ metastasis, blood samples collected on the day when metastasis was confirmed were used; for patients without distant organ metastasis, blood samples collected at last follow-up were used.

After an overnight fast, $3 \mathrm{~mL}$ of peripheral blood was drawn into collection tubes with sodium citrate for the measurement of D-dimer, and $5 \mathrm{~mL}$ of blood was collected into serum-separating tubes for CEA and LDH detection. D-dimer values were determined with a Microparticle Enzyme Immunoassay Analyzer (SYSMEX CS-5100) using the commercially available D-dimer assay kit. CEA was detected with an electrochemiluminescence-based immunoassay analyzer (Roche Cobas e441). Automatic biochemistry analyzer (Roche Cobas 8000) was used to measure LDH level by spectrophotometry. The reference ranges of D-dimer, CEA, and LDH in the study were $(0-0.55 \mathrm{mg} / \mathrm{L} \mathrm{FEU}),(0-5 \mathrm{ng} /$ $\mathrm{mL}$ ), and (115-220 U/L), respectively. 


\section{Statistical analysis}

Statistical analysis was carried out using SPSS software (version 13.0; SPSS Inc., Chicago, IL, USA). Student's $t$-test was used to compare age and tumor sizes of the two patient groups, and the results were presented as mean \pm SD. Wilcoxon rank-sum test was used compare the values of D-dimer, CEA, and LDH between the two patient groups, and results were reported as median and interquartile ranges. Association among clinicopathological variables was determined by chi-squared tests. Kappa identity test was used to examine the agreement between D-dimer and CEA levels, or between D-dimer and LDH levels. The receiver operator characteristic (ROC) curve was used to evaluate the performance of D-dimer, CEA, and combination of D-dimer and CEA in detection of distant organ metastasis. All tests were two-sided, and a $P$-value $<0.05$ was considered statistically significant.

\section{Results}

\section{Patients' characteristics}

Of the 178 patients enrolled, 92 patients with CRC developed distant organ metastasis during follow-up, and 86 patients had no evidence of distant organ metastasis yet at last return visit. No significant difference was found in age, sex, primary tumor site, tumor size, tumor differentiation, and clinical stage between vtwo patient groups, indicating that the two cohorts were comparable. In the metastasis group, 65 patients had hepatic metastasis, 23 had lung metastasis, and four had metastatic lesions in both the liver and lung. Moreover, in eleven metastatic patients, other distant organs were involved, including brain, bone, and spleen. A detailed compilation of these data are presented in Table 1.

In the metastasis group, 51 patients were $\geq 60$ years, 28 had a history of smoking, and 35 received chemotherapy after operation. In contrast, the non-metastasis group comprised 41 patients $\geq 60$ years, 23 patients with a history of smoking, and 31 patients received chemotherapy. The detailed results are summarized in Table 2.

\section{Comparison of D-dimer values between patients who developed distant organ metastasis and those without metachronous metastasis}

Figure 1A shows the distribution of plasma D-dimer levels in CRC patients who developed distant organ metastasis

Table I Demographics and clinical characteristics of the study population

\begin{tabular}{|c|c|c|c|}
\hline Characteristics & Metastasis $(n=92)$ & Non-metastasis $(n=86)$ & $P$-value \\
\hline Age (years) & $59.74 \pm 10.63$ & $58.14 \pm 11.81$ & 0.343 \\
\hline Sex & & & 0.343 \\
\hline Male & 58 & 60 & \\
\hline Female & 34 & 26 & \\
\hline Primary site & & & 0.208 \\
\hline Colon & 45 & 34 & \\
\hline Rectum & 47 & 52 & \\
\hline Tumor size $(\mathrm{cm})$ & $3.83 \pm 2.22$ & $3.72 \pm 1.88$ & 0.733 \\
\hline Differentiation & & & 0.173 \\
\hline Well & 14 & 9 & \\
\hline Middle & 70 & 62 & \\
\hline Poor/mucinous & 8 & 15 & \\
\hline Stage & & & 0.376 \\
\hline 0 & 1 & I & \\
\hline I & 5 & 6 & \\
\hline II & 26 & 34 & \\
\hline III & 60 & 45 & \\
\hline \multicolumn{4}{|l|}{ Metastatic organ } \\
\hline Liver & 65 & - & \\
\hline Lung & 23 & - & \\
\hline Other sites & 11 & - & \\
\hline \multicolumn{4}{|c|}{ Number of involved organs } \\
\hline I & 86 & - & \\
\hline$\geq 2$ & 6 & - & \\
\hline D-dimer (mg/L FEU) & $0.89(0.57,1.27)$ & $0.28(0.18,0.46)$ & $<0.0001$ \\
\hline CEA (ng/mL) & $15.73(3.10,50.28)$ & $2.92(1.94,6.69)$ & $<0.0001$ \\
\hline LDH (U/L) & $192(I 52,230)$ & $161(146,184)$ & $<0.0001$ \\
\hline
\end{tabular}

Note: Values of D-dimer, CEA, and LDH are shown as medians and IQRs.

Abbreviations: CEA, carcinoembryonic antigen; $\mathrm{LDH}$, lactate dehydrogenase. 
Table 2 Comparison of D-dimer levels in CRC patients who developed distant organ metastasis after surgical resection with those in patients without distant organ metastasis based on age, smoking history, and chemotherapy

\begin{tabular}{|c|c|c|c|c|c|}
\hline & \multicolumn{2}{|c|}{ Metastasis } & \multicolumn{2}{|c|}{ Non-metastasis } & \multirow[t]{2}{*}{$P$-value } \\
\hline & $\mathbf{n}$ & $\begin{array}{l}\text { D-dimer value } \\
\text { (mg/L FEU) }\end{array}$ & $\mathbf{n}$ & $\begin{array}{l}\text { D-dimer value } \\
\text { (mg/L FEU) }\end{array}$ & \\
\hline \multicolumn{6}{|l|}{ Age (years) } \\
\hline$<60$ & 41 & $0.72(0.44,1.10)$ & 45 & $0.24(0.17,0.35)$ & $<0.0001$ \\
\hline$\geq 60$ & 51 & $0.7 \mathrm{I}(0.4 \mathrm{I}, \mathrm{I} .34)$ & 41 & $0.31(0.19,0.55)$ & $<0.0001$ \\
\hline \multicolumn{6}{|c|}{ Smoking history } \\
\hline Non-smoker & 64 & $0.72(0.42,1.10)$ & 63 & $0.28(0.18,0.48)$ & $<0.0001$ \\
\hline Smoker & 28 & $0.79(0.4 \mathrm{I}, \mathrm{I} .68)$ & 23 & $0.24(0.20,0.44)$ & 0.0002 \\
\hline \multicolumn{6}{|c|}{ Chemotherapy } \\
\hline No & 57 & $0.68(0.39,1.10)$ & 55 & $0.26(0.16,0.48)$ & $<0.0001$ \\
\hline Yes & 35 & $0.78(0.42,1.23)$ & 31 & $0.28(0.20,0.36)$ & $<0.0001$ \\
\hline
\end{tabular}

Notes: Values of D-dimer are showed as medians and IQRs.

Abbreviation: CRC, colorectal cancer.

("metastasis" in the figures) and those without metachronous metastasis ("non-metastasis" in the figures). The results revealed statistically significant differences between the two groups. As shown in Table 1, the median D-dimer values of patients in the metastasis group and those in the non-metastasis group were $0.89 \mathrm{mg} / \mathrm{L} \mathrm{FEU} \mathrm{and} 0.28 \mathrm{mg} / \mathrm{L}$ FEU, respectively $(P<0.0001)$. With respect to distant organ involvement, plasma D-dimer level was still higher in CRC patients who developed liver metastasis, or other organ metastasis, than in those not demonstrating metastasis (Figure $1 \mathrm{~B}$ and C). Based on the reference range $(0-0.55 \mathrm{mg} / \mathrm{L}$ FEU), $76 \%$ patients (70/92) showed an elevated plasma D-dimer value in the metastasis group, and only $19 \%$ patients (16/86) presented elevated D-dimer values in the non-metastasis group.

The blood samples obtained from patients during followup were checked to determine whether there were still differences in D-dimer levels in every visit of the patients after curative resection. Among $178 \mathrm{CRC}$ patients enrolled, 22 developed distant organ metastasis in their first visit after surgery. In the next four follow-ups, 24, 27, 12, and 7 patients developed distant organ metastasis, respectively. Totally, 92 patients developed distant organ metastasis during five follow-ups. The median interval for occurrence of distant organ metastasis was 8 months (interquartile range 4-11 months). Results showed that the median D-dimer values were elevated in patients who developed distant organ metastasis in each visit (Figure 2). Other details are given in Table 3.

It has been reported that $\mathrm{D}$-dimer concentration increases with age. ${ }^{14,24}$ Additionally, exposure to cigarette smoke and chemotherapy can also cause elevated D-dimer values..$^{25,26}$ To mitigate these variables, patients were divided into two groups based on age, smoking history, and chemotherapy.
The differences in D-dimer values between the two groups were then analyzed. We found that the median D-dimer values were still higher in patients who developed distant organ metastasis than in those without metachronous metastasis regardless of age over or under 60 years. Similar results were also observed in the smoker vs non-smoker group and chemotherapy vs non- chemotherapy group. More details are presented in Figure 3 and Table 2.

\section{Agreement between D-dimer and CEA (or LDH)}

Clinical data showed that CRC patients who developed distant organ metastasis had significantly higher CEA values than those without metachronous metastasis $(15.73 \mathrm{ng} / \mathrm{mL}$ vs 2.92 $\mathrm{ng} / \mathrm{mL}, P<0.0001$ ) (Table 1). Moreover, the serum LDH value was significantly higher in patients with metastasis than in those without metastasis ( $192 \mathrm{U} / \mathrm{L}$ vs $161 \mathrm{U} / \mathrm{L}, P<0.0001)$ (Table 1). According to the reference range mentioned earlier, CEA values were elevated in $72 \%$ patients (66/92) who had developed distant organ metastasis, and LDH values were elevated in $29 \%$ of them (27/92). In the non-metastasis group, $42 \%(36 / 86)$ and $5 \%(4 / 86)$ of patients presented elevated CEA and LDH values, respectively.

Previous studies also reported that elevated CEA and LDH values indicate increased risk for distant organ metastasis in CRC, ${ }^{8,9,27,28}$ so we further investigated the agreement between D-dimer and CEA or LDH values. Table 4 shows that agreement between D-dimer and CEA values was fair ( $\kappa=0.416, P<0.0001)$. In contrast, the identity of D-dimer with LDH was inferior $(\kappa=0.293, P<0.0001)$ (Table 4$)$. Detailed values of D-dimer, CEA, and LDH in CRC patients enrolled in this study are listed in Table S1. 
A

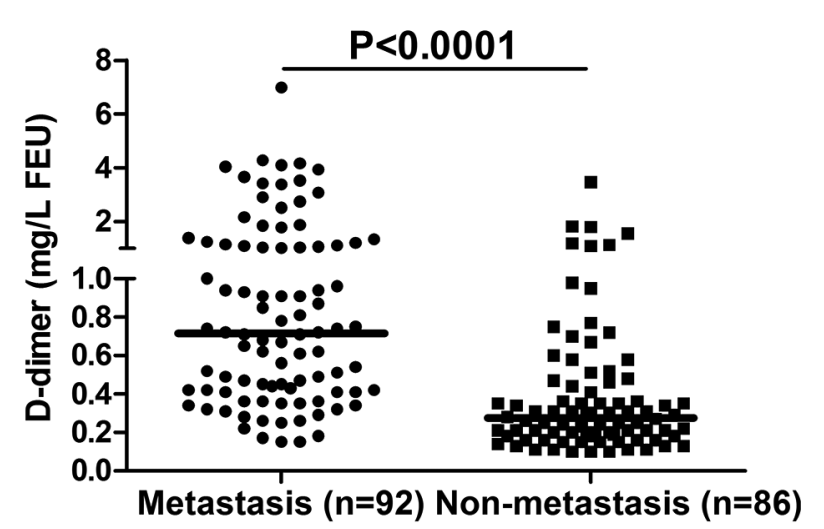

B

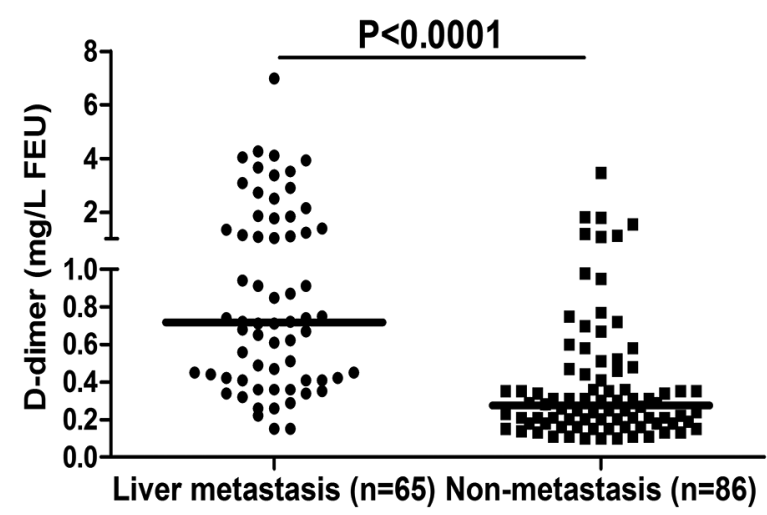

C

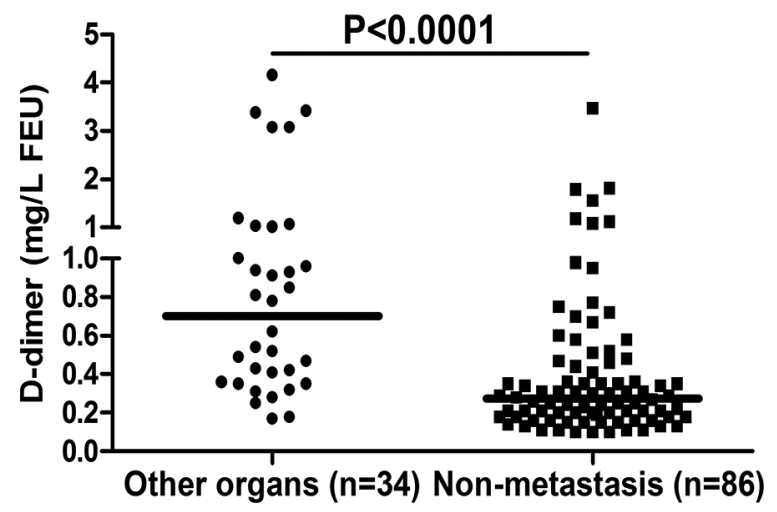

Figure I Dimer levels in colorectal cancer (CRC) patients after curative resection based on the development of distant organ metastasis

Notes: (A) Comparison of D-dimer levels in CRC patients who developed distant organ metastasis with those in patients without distant organ metastasis. (B) Comparison of D-dimer levels in CRC patients who developed liver metastasis with those in patients without distant organ metastasis. (C) Comparison of D-dimer levels in CRC patients who showed other organ involvements (lung, adrenal gland, brain, bone, spleen, and ovary) with those in patients without distant organ metastasis.
Plasma D-dimer value as an indicator for distant organ metastasis in CRC patients after curative resection

As D-dimer values were markedly increased in CRC patients who developed distant organ metastasis, we inferred that plasma D-dimer level could indicate metastases in distant organs.

An ROC curve was used to evaluate the performance of D-dimer, CEA, and LDH on indicating distant organ metastasis in CRC patients after curative resection. The area under the curve (AUC) for D-dimer performance was 0.85 , while the AUC for CEA and LDH was 0.72 and 0.68 , respectively (Figure 4). A reasonable cutoff value of $0.415 \mathrm{mg} / \mathrm{L}$ FEU for D-dimer was determined using the Youden's index. Similarly, the cutoff value for CEA assay was set at $6.095 \mathrm{ng} / \mathrm{mL}$, and the cutoff value for LDH was $208 \mathrm{U} / \mathrm{L}$. Sensitivity, specificity, positive predictive value (PPV), negative predictive value (NPV), and diagnostic accuracy of D-dimer, CEA, LDH, and their combination for indicating distant organ metastasis are summarized in Table 5.

\section{Discussion}

Hypercoagulability and hyperfibrinolysis have been demonstrated in CRC. ${ }^{29}$ Mutant oncogenes, such as $\mathrm{K}$-ras, promote colorectal carcinoma cells to express tissue factor (TF). ${ }^{30,31}$ Once tumor cells detach from primary location and invade into blood vessels, TF on the cytomembrane could initiate the blood coagulation cascades and participate in fibrin formation. Enhanced activity of urokinase-type plasminogen activator (u-PA) has also been demonstrated in colon cancer cells. ${ }^{32} \mathrm{u}$-PA could activate plasminogen and participate in fibrinolysis. Consequently, large quantities of D-dimer are produced due to fibrin degradation. According to the abovementioned studies, D-dimer levels should be elevated when patients develop distant organ metstasis, as more tumor cells have invaded into the circulation. Therefore, we checked the D-dimer levels in the CRC patients who have developed distant organ metstasis after curative resection, and investigated its usefulness for monitoring the development of distant organ metastasis in this study.

Our results revealed that CRC patients who developed distant organ metastasis during follow-up had higher plasma D-dimer levels than those without metachronous metastasis. These results were not affected by patient age, smoking history, or chemotherapy. Moreover, D-dimer level was significantly consistent with CEA level in patients with 
A

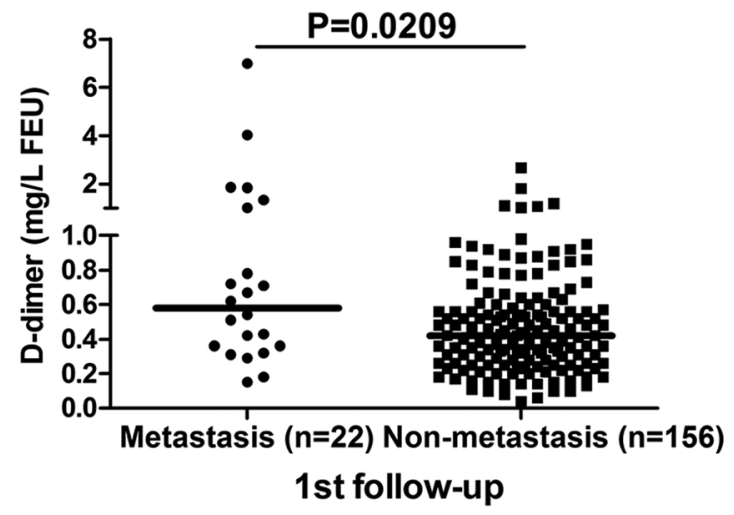

C

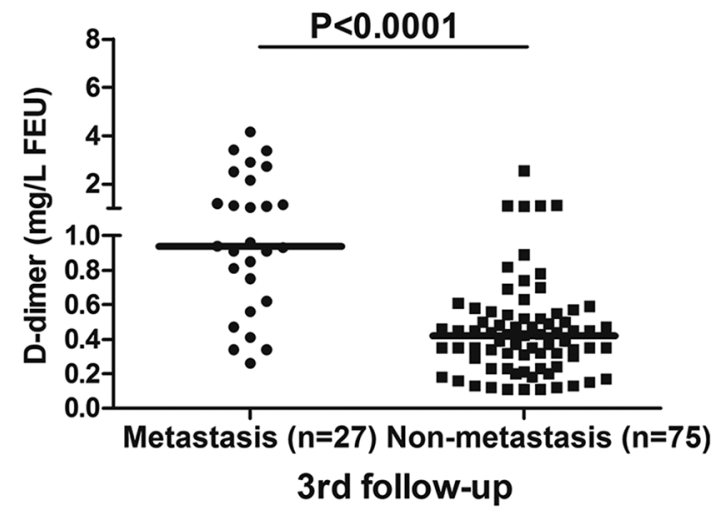

B

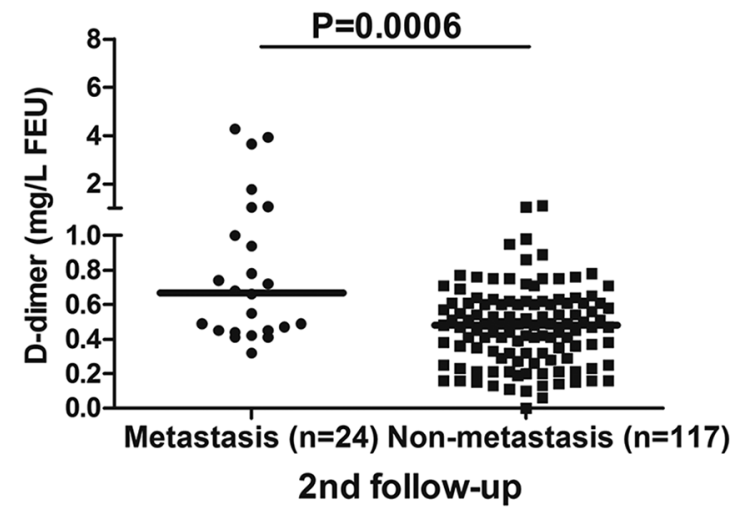

D

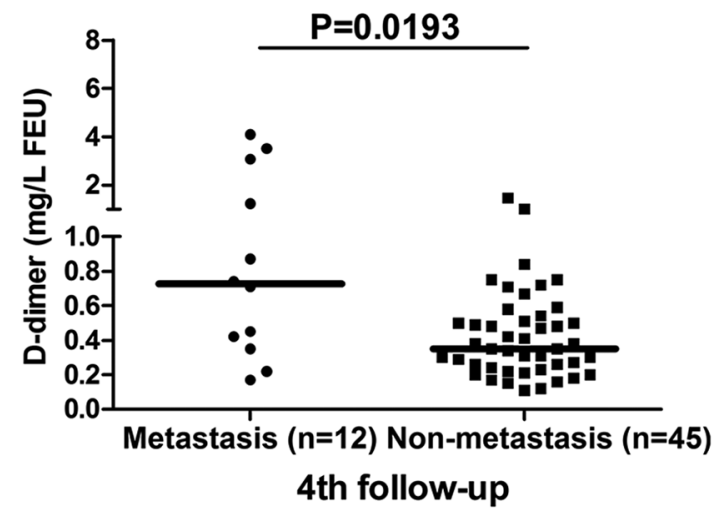

E

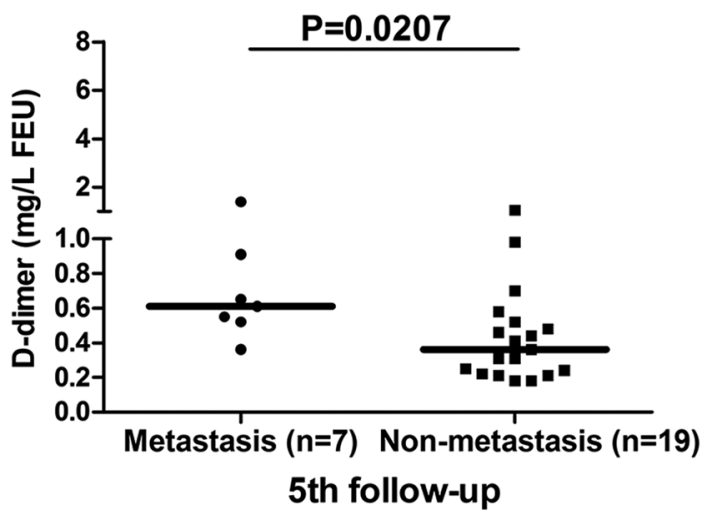

Figure 2 Comparison of D-dimer levels in CRC patients who developed distant organ metastasis with those in patients without distant organ metastasis at different follow-up times.

Notes: (A) The first follow-up, (B) the second follow-up, (C) the third follow-up, (D) the fourth follow-up, and (E) the fifth follow-up. Abbreviation: $\mathrm{CRC}$, colorectal cancer.

CRC. Combining with CEA and/or LDH, D-dimer contributed to detect distant organ metastasis in postoperative CRC patients.
Increased D-dimer level was associated with a shorter survival time, and thus many studies have explored the application of D-dimer as a prognostic marker for CRC. ${ }^{16,22,23}$ 
Table 3 Comparison of D-dimer levels in CRC patients who developed distant organ metastasis after surgical resection with those in patients without distant organ metastasis during follow-up

\begin{tabular}{|l|l|l|l|l|l|}
\hline \multirow{2}{*}{ Follow-up } & \multicolumn{2}{l|}{ Metastasis } & \multicolumn{2}{l|}{ Non-metastasis } & P-value \\
\cline { 2 - 6 } & $\mathbf{n}$ & D-dimer value (mg/L FEU) & $\mathbf{n}$ & D-dimer value (mg/L FEU) & \\
\hline First & 22 & $0.58(0.35,1.10)$ & 156 & $0.42(0.26,0.58)$ & 0.0209 \\
Second & 24 & $0.67(0.45,1.02)$ & 117 & $0.48(0.29,0.61)$ & 0.0006 \\
Third & 27 & $0.94(0.62,2.15)$ & 75 & $0.42(0.24,0.54)$ & $<0.0001$ \\
Fourth & 12 & $0.73(0.37,2.62)$ & 45 & $0.35(0.24,0.53)$ & 0.0193 \\
Fifth & 7 & $0.61(0.52,0.91)$ & 19 & $0.36(0.22,0.52)$ & 0.0207 \\
\hline
\end{tabular}

Notes: Values of D-dimer are showed as medians and IQRs.

Abbreviation: $C R C$, colorectal cancer.

A

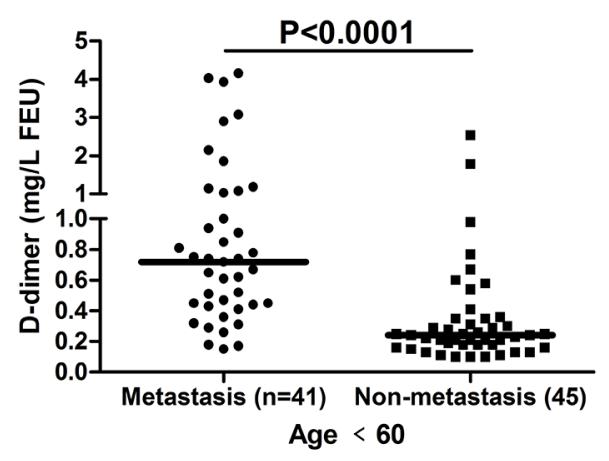

C

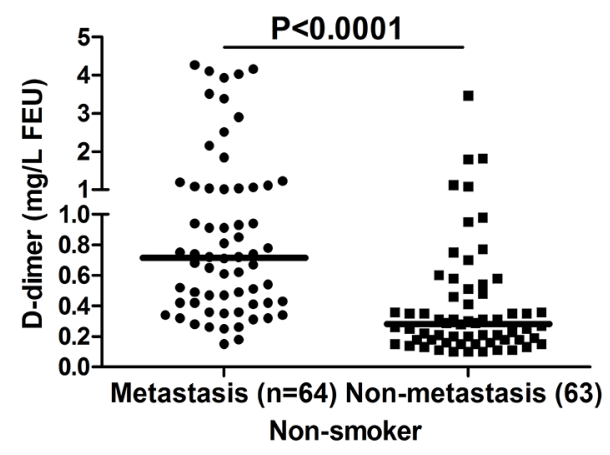

$\mathbf{E}$

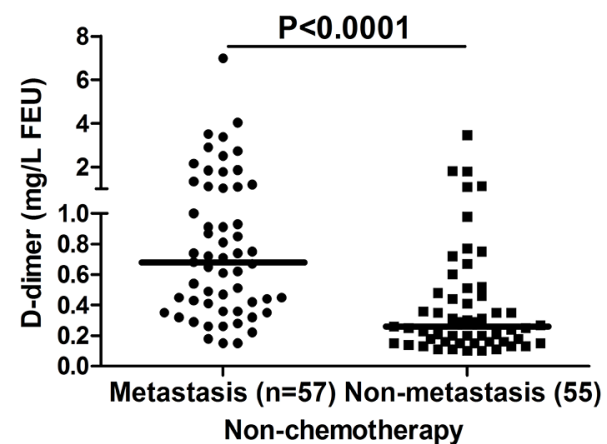

B

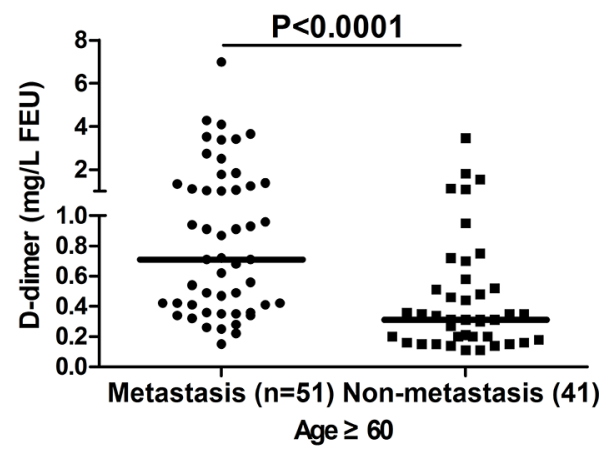

D

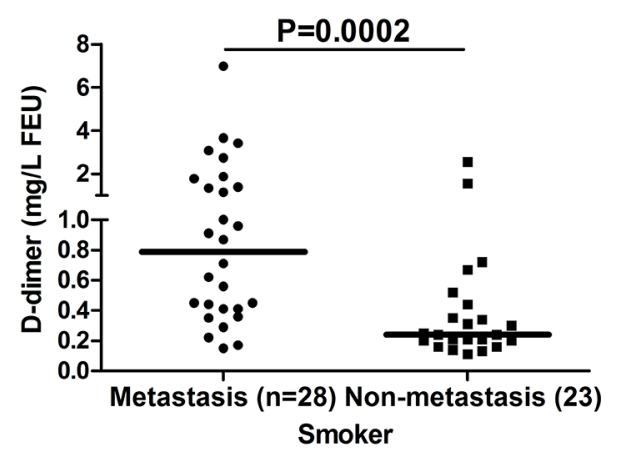

$\mathbf{F}$

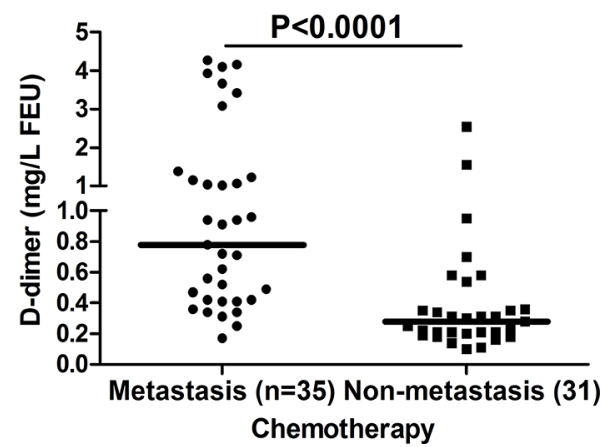

Figure 3 Comparison of D-dimer levels in CRC patients who developed distant organ metastasis after curative resection with those in patients without distant organ metastasis based on age, smoking history, and chemotherapy.

Notes: (A) CRC patients $<60$ years. (B) CRC patients $\geq 60$ years. (C) CRC patients without smoking history. (D) CRC patients with smoking history. (E) CRC patients without chemotherapy. (F) CRC patients with chemotherapy.

Abbreviation: CRC, colorectal cancer. 
Table 4 Agreement between assays in CRC patients

\begin{tabular}{|c|c|c|c|c|c|c|}
\hline \multirow[t]{2}{*}{ D-dimer } & \multicolumn{2}{|c|}{ CEA (ng/mL) } & \multirow[t]{2}{*}{ к } & \multicolumn{2}{|c|}{ LDH (U/L) } & \multirow[t]{2}{*}{ к } \\
\hline & Elevated & Normal & & Elevated & Normal & \\
\hline $\begin{array}{l}\text { Elevated } \\
\text { Normal }\end{array}$ & $\begin{array}{l}63 \\
29\end{array}$ & $\begin{array}{l}23 \\
63\end{array}$ & $\begin{array}{l}0.416 \\
P<0.0001\end{array}$ & $\begin{array}{l}28 \\
3\end{array}$ & $\begin{array}{l}59 \\
88\end{array}$ & $\begin{array}{l}0.293 \\
P<0.0001\end{array}$ \\
\hline
\end{tabular}

Notes: Elevated: above the upper limit of reference range; Normal: within the reference range. Reference range: D-dimer: 0-0.55 mg/L FEU; CEA: 0-5 ng/mL; LDH: I I5-220 U/L.

Abbreviations: CRC, colorectal cancer; CEA, carcinoembryonic antigen; LDH, lactate dehydrogenase.

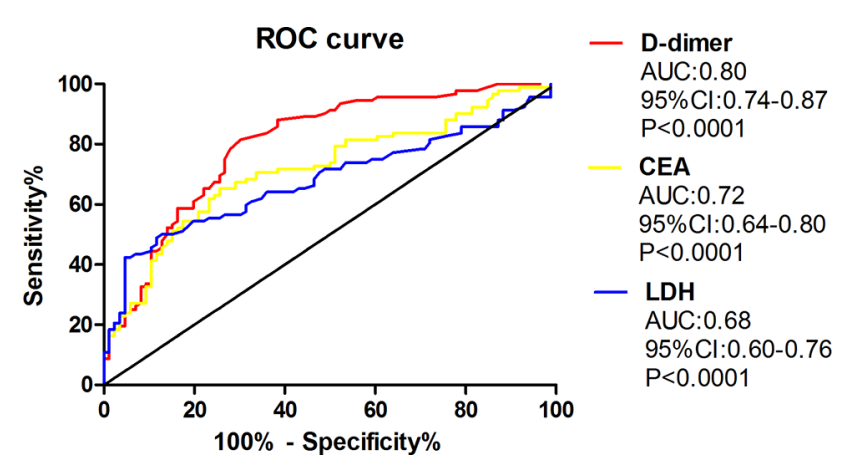

Figure 4 ROC used for detecting distant organ metastasis in CRC patients after curative resection.

Abbreviations: CRC, colorectal cancer; ROC, receiver operator characteristic; $A \cup C$, area under ROC curve; CEA, carcinoembryonic antigen; LDH, lactate dehydrogenase.

However, the relationship between plasma D-dimer and distant organ metastasis was seldom reported. Kilic et al $^{17}$ enrolled $54 \mathrm{CRC}$ patients with synchronous metastasis, and found that there was a difference in D-dimer values between the metastatic positive group and the metastatic negative group before chemotherapy (373.7 IU/mL vs $603 \mathrm{IU} / \mathrm{mL}$ ). Another study demonstrated that preoperative D-dimer level in patients with Dukes D CRC was significantly higher than in those with Dukes A, B, or C cancer. ${ }^{10}$ In the study mentioned above, 23 patients with Dukes D stage underwent operation (exploratory laparotomy) for diagnosis intent, and all were confirmed as having synchronous metastasis. By comparison, our study revealed the first observation that plasma D-dimer level is higher in CRC patients who developed distant organ metastasis after curative resection of the primary tumor.

Presently, the most common tumor marker applied to assess the risk of metachronous metastasis in CRC is serum CEA. ${ }^{33,34} \mathrm{LDH}$ is another reflector of tumor burden, and linked to distant organ metastasis in CRC. ${ }^{9,17}$ Similarly, distributions of CEA and LDH were both markedly higher among CRC patients with metachronous metastasis in our study. Moreover, we found that D-dimer rise was fairly consistent with higher CEA, suggesting that elevated D-dimer level might also indicate the occurrence of distant organ metastasis in patients with resected CRC. However, the agreement of D-dimer with LDH was relatively low. The reason might be that most values of LDH still fell within the reference range in the metastasis group, although they were elevated compared to those in non-metastasis patients.

CEA is recommended as a biomarker for predicting tumor recurrence for CRC in the guidelines issued by the National Comprehensive Cancer Network in 2017. ${ }^{33,34}$ Elevated LDH levels also indicate an increased risk for metachronous metastasis in CRC. ${ }^{9}$ Therefore, we compared the performance of D-dimer level in detecting metachronous metastasis with that of CEA or LDH. The results of ROC curve showed that AUC of D-dimer assay $(0.8,95 \% \mathrm{CI}$ : $0.74-0.87)$ is larger than that of CEA $(0.72$, 95\%CI: $0.64-0.80)$ or $\mathrm{LDH}(0.68,95 \% \mathrm{CI}$ : 0.60-0.76), indicating that D-dimer had the advantage over CEA or LDH in differentiating patients who developed distant organ metastasis from CRC after undergoing primary tumor resection. The specificity of D-dimer alone $(73.3 \%)$ was lower than that of CEA alone (74.4\%), but the sensitivity (88.0\%), PPV (77.9\%), NPV (85.1\%), and accuracy $(80.9 \%$ vs $69.7 \%$ ) in D-dimer assay were superior to those in CEA assay $(65.2 \%, 73.2 \%$ and $80.9 \%$, respectively). Other studies also reported that CEA assay had a moderate sensitivity and specificity for distant organ metastasis in patients with $\mathrm{CRC},{ }^{7,10,17}$ mainly because CEA is relatively insensitive to nonliver metastasis. ${ }^{27,35}$ By comparison, LDH showed the lowest sensitivity (42.4\%) and highest specificity (95.3\%) among the three biomarkers. Dong et $\mathrm{al}^{36}$ found that the sensitivity of LDH for predicting brain metastasis in triple-negative breast cancer was $44.0 \%$, which was similar to our result. Different combinations could further improve diagnostic performance. The sensitivity and NPV of a combination assay (either D-dimer elevation or CEA elevation) increased to 94.6\% and 91.1\%, respectively, and the specificity and PPV of another combination assay (both D-dimer elevation and LDH elevation) were $97.7 \%$ and $94.9 \%$, respectively. Parallel test of the three markers increased the sensitivity and NPV to $95.7 \%$ and $92.7 \%$, respectively. The results suggested that the combination of serum/plasma biomarkers could be warning 
Table 5 Sensitivity, specificity, PPV, NPV, and diagnostic accuracy of D-dimer, CEA, LDH, and combinations of D-dimer with CEA and $\mathrm{LDH}$ for detecting distant organ metastasis in CRC patients after curative resection

\begin{tabular}{|l|l|l|l|l|l|}
\hline & Sensitivity & Specificity & PPV & NPV & Accuracy \\
\hline D-dimer alone & 88.0 & 73.3 & 77.9 & 85.1 & 80.9 \\
CEA alone & 65.2 & 74.4 & 73.2 & 66.7 & 69.7 \\
LDH alone & 42.4 & 95.3 & 90.7 & 60.7 & 68.0 \\
D-dimer or CEA & 94.6 & 59.3 & 71.3 & 91.1 & 77.5 \\
D-dimer and CEA & 56.5 & 87.2 & 82.5 & 65.2 & 71.3 \\
D-dimer or LDH & 90.2 & 70.9 & 76.9 & 87.1 & 80.9 \\
D-dimer and LDH & 40.2 & 97.7 & 94.9 & 60.4 & 68.0 \\
Serial test & 29.3 & 96.5 & 90.0 & 56.1 & 61.8 \\
Parallel test & 95.7 & 59.3 & 71.5 & 92.7 & 78.1 \\
\hline
\end{tabular}

Notes: The cutoff value of D-dimer is $0.415 \mathrm{mg} / \mathrm{mL} \mathrm{FEU,} \mathrm{the} \mathrm{cutoff} \mathrm{value} \mathrm{of} \mathrm{CEA} \mathrm{is} 6.095 \mathrm{ng} / \mathrm{mL}$, and the cutoff value of LDH is $208 \mathrm{U} / \mathrm{L}$. Values show the percentages. Serial tests: distant organ metastasis was determined only when the values of D-dimer, CEA, and LDH were all higher than cutoff values. Parallel tests: distant organ metastasis was determined as long as one of D-dimer value, CEA value, and LDH value was higher than cutoff value(s).

Abbreviations: CEA, carcinoembryonic antigen; CRC, colorectal cancer; LDH, lactate dehydrogenase; NPV, negative predictive value; PPV, positive predictive value.

indicators for detailed imaging examination. In general, CRC patients are followed up routinely at 2-month intervals for the first 2 years and at 6-month intervals thereafter. At each visit, blood samples are collected. However, chest radiographs and abdominopelvic CT are only performed 6 months postoperatively and then at yearly intervals. Therefore, if levels of D-dimer, CEA, or LDH are elevated in a CRC patient after curative resection during a return visit, timely imaging examination for distant organ metastasis should be recommended.

There surely are some potential limitations in the study. Firstly, patients with other cancers could also show elevated D-dimer level, so this feature is not an exclusive indicator for distant organ metastasis only in patients with CRC. Secondly, relevance between $\mathrm{D}$-dimer values and distant organ metastasis in CRC still requires further validation with prospective trials.

\section{Conclusion}

Our study demonstrated that D-dimer level was elevated in postoperative CRC patients who developed distant organ metastasis. Combining with CEA and/or LDH, D-dimer could be a useful surveillance marker for distant organ metastasis in CRC after curative resection.

\section{Acknowledgments}

This work was supported by the CAMS Innovation Fund for Medical Sciences (CIFMS) (Grant No 2017-I2M-3-005), the National Natural Science Foundation of China (Grant No 81778272) and Beijing Hope Run Special Fund of Cancer Foundation of China (LC2015B13).

\section{Author contributions}

YG contributed to the study design, data collection and analysis, and wrote the main manuscript text; FC conceived the study and contributed to statistical analysis; WC revised the manuscript and funded the study. All authors contributed toward data analysis, drafting and critically revising the paper and agree to be accountable for all aspects of the work.

\section{Disclosure}

The authors report no conflicts of interest in this work.

\section{References}

1. Ferlay J, Soerjomataram I, Dikshit R, et al. Cancer incidence and mortality worldwide: sources, methods and major patterns in GLOBOCAN 2012. Int J Cancer. 2015;136(5):E359-E386.

2. Fang JY, Dong HL, Sang XJ, et al. Colorectal Cancer Mortality Characteristics and Predictions in China, 1991-2011. Asian Pac J Cancer Prev. 2015;16(17):7991-7995.

3. Chuang SC, Su YC, Lu CY, et al. Risk factors for the development of metachronous liver metastasis in colorectal cancer patients after curative resection. World J Surg. 2011;35(2):424-429.

4. Kim CH, Huh JW, Kim HJ, et al. Factors influencing oncological outcomes in patients who develop pulmonary metastases after curative resection of colorectal cancer. Dis Colon Rectum. 2012;55(4): 459-464.

5. Memon AA, Sundquist K, Pirouzifard M, et al. Identification of novel diagnostic biomarkers for deep venous thrombosis. $\mathrm{Br} J$ Haematol. 2018;181(3):378-385.

6. Tsurusaki M, Sofue K, Murakami T. Current evidence for the diagnostic value of gadoxetic acid-enhanced magnetic resonance imaging for liver metastasis. Hepatol Res. 2016;46(9):853-861.

7. Su BB, Shi H, Wan J. Role of serum carcinoembryonic antigen in the detection of colorectal cancer before and after surgical resection. World J Gastroenterol. 2012;18(17):2121-2126.

8. Laubert T, Bente V, Freitag-Wolf S, et al. Aneuploidy and elevated CEA indicate an increased risk for metachronous metastasis in colorectal cancer. Int J Colorectal Dis. 2013;28(6):767-775.

9. Wu XZ, Ma F, Wang XL. Serological diagnostic factors for liver metastasis in patients with colorectal cancer. World J Gastroenterol. 2010;16(32):4084-4088.

10. Oya M, Akiyama Y, Yanagida T, Akao S, Ishikawa H. Plasma D-dimer level in patients with colorectal cancer: its role as a tumor marker. Surg Today. 1998;28(4):373-378.

11. Flamini E, Mercatali L, Nanni O, et al. Free DNA and carcinoembryonic antigen serum levels: an important combination for diagnosis of colorectal cancer. Clin Cancer Res. 2006;12(23):6985-6988. 
12. Kawai K, Watanabe T. Colorectal cancer and hypercoagulability. Surg Today. 2014;44(5):797-803.

13. Edwards CM, Warren J, Armstrong L, Donnelly PK. D-dimer: a useful marker of disease stage in surgery for colorectal cancer. Br J Surg. 1993;80(11):1404-1405.

14. Oya M, Akiyama Y, Okuyama T, Ishikawa H. High preoperative plasma D-dimer level is associated with advanced tumor stage and short survival after curative resection in patients with colorectal cancer. Jpn J Clin Oncol. 2001;31(8):388-394.

15. Xu G, Zhang YL, Huang W. Relationship between plasma D-dimer levels and clinicopathologic parameters in resectable colorectal cancer patients. World J Gastroenterol. 2004;10(6):922-923.

16. Zhu L, Liu B, Zhao Y, et al. High levels of D-dimer correlated with disease status and poor prognosis of inoperable metastatic colorectal cancer patients treated with bevacizumab. $J$ Cancer Res Ther. 2014;10(8): 246-251.

17. Kilic L, Yildiz I, Sen FK, et al. D-dimer and international normalized ratio (INR) are correlated with tumor markers and disease stage in colorectal cancer patients. Cancer Biomark. 2015;15(4):405-411.

18. Tekeşin K, Bayrak S, Esatoğlu V, Özdemir E, Özel L, Melih Kara V. D-Dimer and Carcinoembryonic Antigen Levels: Useful Indicators for Predicting the Tumor Stage and Postoperative Survival. Gastroenterol Res Pract. 2016;2016:4295029.

19. Blackwell K, Hurwitz H, Liebérman G, et al. Circulating D-dimer levels are better predictors of overall survival and disease progression than carcinoembryonic antigen levels in patients with metastatic colorectal carcinoma. Cancer. 2004;101(1):77-82.

20. Kilic M, Yoldas O, Keskek M, et al. Prognostic value of plasma D-dimer levels in patients with colorectal cancer. Colorectal Dis. 2008;10(3):238-241.

21. Stender MT, Larsen TB, Sørensen HT, Thorlacius-Ussing O. Preoperative plasma D-dimer predicts 1-year survival in colorectal cancer patients with absence of venous thromboembolism (VTE): a prospective clinical cohort study. J Thromb Haemost. 2012;10(10):2027-2031.

22. Yamamoto M, Yoshinaga K, Matsuyama A, et al. Plasma D-dimer level as a mortality predictor in patients with advanced or recurrent colorectal cancer. Oncology. 2012;83(1):10-15.

23. Motavaf E, Sunesen KG, Stender MT, Thorlacius-Ussing O. Prognostic value of preoperative $\mathrm{D}$-dimer and carcinoembryonic antigen levels in patients undergoing intended curative resection for colorectal cancer: a prospective cohort study. Int J Colorectal Dis. 2014;29(11):1427-1432.

24. Harper PL, Theakston E, Ahmed J, Ockelford P. D-dimer concentration increases with age reducing the clinical value of the D-dimer assay in the elderly. Intern Med J. 2007;37(9):607-613.
25. Caponnetto P, Russo C, di Maria A, et al. Circulating endothelialcoagulative activation markers after smoking cessation: a 12-month observational study. Eur J Clin Invest. 2011;41(6):616-626.

26. di Nisio M, Ferrante N, de Tursi M, et al. Incidental venous thromboembolism in ambulatory cancer patients receiving chemotherapy. Thromb Haemost. 2010;104(5):1049-1054.

27. Pakdel A, Malekzadeh M, Naghibalhossaini F. The association between preoperative serum CEA concentrations and synchronous liver metastasis in colorectal cancer patients. Cancer Biomark. 2016;16(2): $245-252$.

28. Koukourakis MI, Giatromanolaki A, Simopoulos C, Polychronidis A, Sivridis E. Lactate dehydrogenase 5 (LDH5) relates to up-regulated hypoxia inducible factor pathway and metastasis in colorectal cancer. Clin Exp Metastasis. 2005;22(1):25-30.

29. Falanga A, Marchetti M, Vignoli A. Coagulation and cancer: biological and clinical aspects. J Thromb Haemost. 2013;11(2):223-233.

30. Shirasawa S, Furuse M, Yokoyama N, Sasazuki T. Altered growth of human colon cancer cell lines disrupted at activated Ki-ras. Science. 1993;260(5104):85-88.

31. Yu JL, May L, Lhotak V, et al. Oncogenic events regulate tissue factor expression in colorectal cancer cells: implications for tumor progression and angiogenesis. Blood. 2005;105(4):1734-1741.

32. Harvey SR, Sait SN, Xu Y, Bailey JL, Penetrante RM, Markus G. Demonstration of urokinase expression in cancer cells of colon adenocarcinomas by immunohistochemistry and in situ hybridization. Am J Pathol. 1999;155(4):1115-1120.

33. National Comprehensive Cancer Network (NCCN), Clinical Practice Guidelines in Oncology, Colon Cancer, Version 2; 2017. Available from: https://www.nccn.org/professionals/physician_gls/pdf/colon.pdf. Accessed March 13, 2017.

34. National Comprehensive Cancer Network (NCCN), Clinical Practice Guidelines in Oncology, Recral Cancer, Version 3; 2017. Available from: https://www.nccn.org/professionals/physician_gls/pdf/rectal.pdf. Accessed March 13, 2017.

35. Moertel CG, Fleming TR, Macdonald JS, Haller DG, Laurie JA, Tangen C. An evaluation of the carcinoembryonic antigen (CEA) test for monitoring patients with resected colon cancer. JAMA. 1993;270(8): 943-947.

36. Dong T, Liu Z, Xuan Q, Wang Z, Ma W, Zhang Q. Tumor LDH-A expression and serum LDH status are two metabolic predictors for triple negative breast cancer brain metastasis. Sci Rep. 2017;7(1):6069. 


\section{Supplementary material}

Table SI Values of D-dimer, CEA, and LDH in CRC patients in the study based on the reference range

\begin{tabular}{|c|c|c|c|c|c|c|}
\hline \multirow[t]{3}{*}{ Patient No } & \multicolumn{2}{|c|}{ D-dimer (mg/L FEU) } & \multicolumn{2}{|c|}{ CEA (ng/mL) } & \multicolumn{2}{|c|}{ LDH (U/L) } \\
\hline & $\leq 0.55$ & $>0.55$ & $\leq 5$ & $>5$ & $\leq \mathbf{2 2 0}$ & $>\mathbf{2 2 0}$ \\
\hline & $n=91$ & $n=87$ & $n=85$ & $n=93$ & $n=145$ & $n=33$ \\
\hline 1 & & 0.78 & & 23.71 & 175 & \\
\hline 2 & & 0.56 & & 177.7 & & 522 \\
\hline 3 & & 1.86 & & 84.2 & & 245 \\
\hline 4 & & 0.71 & & 34.6 & 159 & \\
\hline 5 & & 4.16 & & 17.99 & 146 & \\
\hline 6 & 0.28 & & & 198.3 & & 225 \\
\hline 7 & 0.49 & & 14.36 & & 166 & \\
\hline 8 & & 2.9 & 1.24 & & 190 & \\
\hline 9 & & 0.91 & & 932 & & 1149 \\
\hline 10 & & 0.62 & & $87 I .4$ & 132 & \\
\hline II & 0.32 & & 3.12 & & 132 & \\
\hline 12 & & 3.93 & & 2389 & & 783 \\
\hline 13 & & 1.08 & & 5.03 & 119 & \\
\hline 14 & 0.35 & & & 21.69 & 164 & \\
\hline 15 & & 1.78 & 3.6 & & 219 & \\
\hline 16 & 0.45 & & 1.67 & & 150 & \\
\hline 17 & & 0.75 & 0.95 & & & 265 \\
\hline 18 & & 0.74 & & 6.17 & 139 & \\
\hline 19 & & 4.03 & & 304.2 & & 270 \\
\hline 20 & 0.36 & & & 12.36 & 143 & \\
\hline 21 & & 4.27 & 0.57 & & 167 & \\
\hline 22 & 0.47 & & 1.34 & & 210 & \\
\hline 23 & & 0.72 & & 506.2 & & 237 \\
\hline 24 & & $\mathrm{I}$ & & 146.7 & & 652 \\
\hline 25 & & 0.62 & & 8.69 & & 651 \\
\hline 26 & 0.26 & & & 134.9 & 148 & \\
\hline 27 & & 2.15 & & 24.3 & 209 & \\
\hline 28 & 0.18 & & 1.5 & & 170 & \\
\hline 29 & & 1.01 & & 75.59 & 144 & \\
\hline 30 & 0.43 & & & 11.23 & 110 & \\
\hline 31 & & 1.03 & 4.92 & & 166 & \\
\hline 32 & & 3.08 & & 214.6 & & 772 \\
\hline 33 & & 4.1 & & 645.3 & 209 & \\
\hline 34 & 0.15 & & 1.82 & & 137 & \\
\hline 35 & & 1.03 & 2.85 & & & 444 \\
\hline 36 & 0.26 & & & 38.54 & 179 & \\
\hline 37 & & 0.71 & & 7.76 & & 221 \\
\hline 38 & & 2.59 & & 5.23 & & 258 \\
\hline 39 & & 1.13 & & 19.33 & 133 & \\
\hline 40 & & 1.28 & & 72.4 & & 252 \\
\hline $4 I$ & & 2.74 & & 17.59 & & 271 \\
\hline 42 & & 1.05 & & 35.19 & 177 & \\
\hline 43 & & 0.91 & & 7.24 & & 224 \\
\hline 44 & & 1.84 & & 3393 & & 683 \\
\hline 45 & & 0.60 & & 68.67 & & 230 \\
\hline 46 & & 0.74 & & 6.17 & 139 & \\
\hline 47 & & 0.61 & & 28 & 145 & \\
\hline 48 & & 1.03 & 1.93 & & & 234 \\
\hline 49 & & 1.06 & 2.99 & & 153 & \\
\hline 50 & & 0.93 & 1.25 & & 102 & \\
\hline 51 & & 1.64 & 1.67 & & 183 & \\
\hline
\end{tabular}


Table SI (Continued)

\begin{tabular}{|c|c|c|c|c|c|c|}
\hline \multirow[t]{3}{*}{ Patient No } & \multicolumn{2}{|c|}{ D-dimer (mg/L FEU) } & \multicolumn{2}{|c|}{ CEA (ng/mL) } & \multicolumn{2}{|c|}{ LDH (U/L) } \\
\hline & $\leq 0.55$ & $>0.55$ & $\leq 5$ & $>5$ & $\leq \mathbf{2 2 0}$ & $>\mathbf{2 2 0}$ \\
\hline & $n=91$ & $n=87$ & $n=85$ & $n=93$ & $n=145$ & $n=33$ \\
\hline 52 & 0.42 & & 2.39 & & 176 & \\
\hline 53 & 0.52 & & & 17.6 & 106 & \\
\hline 54 & & 1.04 & & 5.42 & 202 & \\
\hline 55 & & 0.63 & 4.19 & & 215 & \\
\hline 56 & & 1.02 & & 26.18 & 163 & \\
\hline 57 & & 1.8 & & 16.25 & $15 \mid$ & \\
\hline 58 & & 3.66 & & 12.71 & & 229 \\
\hline 59 & & $3.5 I$ & & 2147 & & 297 \\
\hline 60 & & 0.94 & 2.61 & & 187 & \\
\hline 61 & & 1.15 & & 1626 & 204 & \\
\hline 62 & & 0.72 & 3.09 & & 191 & \\
\hline 63 & & 0.61 & & 10.6 & 194 & \\
\hline 64 & 0.55 & & 2.82 & & 211 & \\
\hline 65 & & 1.78 & & 468.3 & 175 & \\
\hline 66 & & 3.42 & & 15.52 & 179 & \\
\hline 67 & 0.42 & & & 11.08 & 193 & \\
\hline 68 & & 0.67 & & \begin{tabular}{|l|}
48.43 \\
\end{tabular} & & 235 \\
\hline 69 & & 0.96 & & $39.5 \mathrm{I}$ & 214 & \\
\hline 70 & & 0.69 & & 53.73 & 212 & \\
\hline 71 & & 1.19 & & \begin{tabular}{|l|}
24.27 \\
\end{tabular} & 137 & \\
\hline 72 & 0.22 & & & \begin{tabular}{|l|}
50.89 \\
\end{tabular} & & 221 \\
\hline 73 & & 0.68 & & 35.19 & 159 & \\
\hline 74 & & 3.07 & 1.48 & & 132 & \\
\hline 75 & 0.25 & & 3.15 & & 190 & \\
\hline 76 & & 0.91 & 1.19 & & 137 & \\
\hline 77 & & 0.73 & & 8.69 & 147 & \\
\hline 78 & & 6.98 & & 17.07 & & 242 \\
\hline 79 & & 0.68 & & 8.06 & 201 & \\
\hline 80 & & 1.07 & & 5.69 & 148 & \\
\hline 81 & 0.29 & & & 44.93 & 180 & \\
\hline 82 & & 0.85 & & 90.86 & & 234 \\
\hline 83 & & 0.75 & & 62.31 & 212 & \\
\hline 84 & 0.49 & & 3.04 & & 216 & \\
\hline 85 & & 1.23 & & 15.94 & & 222 \\
\hline 86 & & 0.87 & & 20.36 & 190 & \\
\hline 87 & & 1.11 & 3.06 & & 194 & \\
\hline 88 & & 1.34 & 1.90 & & 164 & \\
\hline 89 & 0.42 & & 1.93 & & & 237 \\
\hline 90 & & 0.61 & & 30.44 & & 455 \\
\hline 91 & 0.54 & & & 22.83 & 197 & \\
\hline 92 & & 0.94 & 1.35 & & 151 & \\
\hline 93 & 0.15 & & & 21.61 & 176 & \\
\hline 94 & 0.30 & & & 15.31 & 181 & \\
\hline 95 & 0.47 & & 3.66 & & 185 & \\
\hline 96 & & 0.67 & 2.06 & & 130 & \\
\hline 97 & 0.16 & & 1.62 & & 167 & \\
\hline 98 & 0.26 & & 3.51 & & 160 & \\
\hline 99 & 0.20 & & & \begin{tabular}{|l|}
42.75 \\
\end{tabular} & 146 & \\
\hline 100 & & 0.98 & & \begin{tabular}{|l|}
236.8 \\
\end{tabular} & 132 & \\
\hline 101 & 0.11 & & 1.39 & & 160 & \\
\hline 102 & 0.36 & & 2.09 & & 151 & \\
\hline 103 & & 1.12 & & 13.08 & 184 & \\
\hline 104 & 0.24 & & 0.82 & & 133 & \\
\hline 105 & & 0.95 & 0.78 & & 191 & \\
\hline
\end{tabular}


Table SI (Continued)

\begin{tabular}{|c|c|c|c|c|c|c|}
\hline \multirow[t]{3}{*}{ Patient No } & \multicolumn{2}{|c|}{ D-dimer (mg/L FEU) } & \multicolumn{2}{|c|}{ CEA (ng/mL) } & \multicolumn{2}{|c|}{ LDH (U/L) } \\
\hline & $\leq 0.55$ & $>0.55$ & $\leq 5$ & $>5$ & $\leq 220$ & $>220$ \\
\hline & $n=91$ & $n=87$ & $n=85$ & $n=93$ & $n=145$ & $n=33$ \\
\hline 106 & & 1.79 & & 43.62 & & 232 \\
\hline 107 & & 0.75 & 1.09 & & 182 & \\
\hline 108 & 0.31 & & & 5.82 & 130 & \\
\hline 109 & 0.21 & & 0.69 & & 154 & \\
\hline 110 & 0.11 & & 2.66 & & 142 & \\
\hline 111 & & 0.58 & 4.54 & & 151 & \\
\hline 112 & 0.15 & & 1.68 & & 189 & \\
\hline 113 & 0.16 & & 2.41 & & 171 & \\
\hline 114 & & 0.72 & & 10.32 & 172 & \\
\hline 115 & 0.20 & & 1.95 & & 146 & \\
\hline 116 & 0.19 & & 1.66 & & 163 & \\
\hline 117 & 0.29 & & 0.99 & & 169 & \\
\hline 118 & 0.16 & & 1.25 & & 153 & \\
\hline 119 & 0.20 & & 2.02 & & 129 & \\
\hline 120 & & 0.60 & & 12.28 & 204 & \\
\hline 121 & 0.48 & & 1.94 & & 162 & \\
\hline 122 & 0.10 & & 2.15 & & 177 & \\
\hline 123 & 0.25 & & 2.89 & & 136 & \\
\hline 124 & 0.31 & & & 19.04 & 158 & \\
\hline 125 & 0.11 & & 1.46 & & 142 & \\
\hline 126 & 0.30 & & 2.73 & & 159 & \\
\hline 127 & 0.35 & & & 11.75 & 191 & \\
\hline 128 & 0.10 & & 1.56 & & 136 & \\
\hline 129 & 0.15 & & 3.27 & & 145 & \\
\hline 130 & 0.14 & & 0.74 & & 150 & \\
\hline 131 & 0.34 & & & 11.34 & 170 & \\
\hline 132 & 0.46 & & & 41.14 & 145 & \\
\hline 133 & 0.23 & & & 124.8 & 128 & \\
\hline 134 & 0.36 & & & 6.02 & 148 & \\
\hline 135 & & \begin{tabular}{|l|}
3.47 \\
\end{tabular} & & 80.20 & & 285 \\
\hline 136 & & 1.08 & & 5.39 & 183 & \\
\hline 137 & 0.35 & 3.78 & & & 202 & \\
\hline 138 & 0.20 & & & 5.85 & 146 & \\
\hline 139 & & 0.70 & & 6.43 & 173 & \\
\hline 140 & 0.31 & & 0.46 & & 190 & \\
\hline $14 \mid$ & 0.21 & & 1.78 & & 142 & \\
\hline 142 & 0.10 & & & 53.95 & 192 & \\
\hline 143 & 0.52 & & 2.94 & & 197 & \\
\hline 144 & 0.29 & & 2.62 & & 140 & \\
\hline 145 & 0.15 & & 2.79 & & 203 & \\
\hline 146 & 0.18 & & 4.50 & & 133 & \\
\hline 147 & 0.35 & & 3.64 & & 167 & \\
\hline 148 & 0.31 & & 0.85 & & 150 & \\
\hline 149 & 0.28 & & 4.38 & & 149 & \\
\hline 150 & $0.5 I$ & & 3.87 & & 150 & \\
\hline 151 & 0.22 & & 1.02 & & 181 & \\
\hline 152 & 0.34 & & 1.21 & & 188 & \\
\hline 153 & 0.21 & & 2.43 & & 145 & \\
\hline 154 & & 0.58 & & \begin{tabular}{|l|}
7.48 \\
\end{tabular} & 154 & \\
\hline 155 & 0.21 & & 3.31 & & 152 & \\
\hline 156 & & 1.18 & & 52.24 & 207 & \\
\hline 157 & & 1.55 & 3.7 & & 190 & \\
\hline 158 & 0.25 & & & \begin{tabular}{|l|}
5.08 \\
\end{tabular} & 140 & \\
\hline 159 & & 0.77 & 0.68 & & 164 & \\
\hline
\end{tabular}


Table SI (Continued)

\begin{tabular}{|c|c|c|c|c|c|c|}
\hline \multirow[t]{3}{*}{ Patient No } & \multicolumn{2}{|c|}{ D-dimer (mg/L FEU) } & \multicolumn{2}{|c|}{ CEA (ng/mL) } & \multicolumn{2}{|c|}{ LDH (U/L) } \\
\hline & $\leq 0.55$ & $>0.55$ & $\leq 5$ & $>5$ & $\leq 220$ & $>220$ \\
\hline & $n=91$ & $n=87$ & $n=85$ & $n=93$ & $n=145$ & $n=33$ \\
\hline 160 & 0.44 & & 2.25 & & 180 & \\
\hline 161 & 0.13 & & & 5.10 & 156 & \\
\hline 162 & 0.13 & & & 5.25 & 193 & \\
\hline 163 & 0.25 & & 4.15 & & 187 & \\
\hline 164 & 0.21 & & 2.24 & & 150 & \\
\hline 165 & 0.21 & & & 9.30 & 171 & \\
\hline 166 & 0.18 & & 2.35 & & 202 & \\
\hline 167 & 0.35 & & 1.38 & & 150 & \\
\hline 168 & 0.11 & & 2.67 & & & 236 \\
\hline 169 & 0.35 & & 2.09 & & 152 & \\
\hline 170 & $0.4 I$ & & 3.13 & & 175 & \\
\hline 171 & & 1.81 & 1.05 & & 141 & \\
\hline 172 & 0.16 & & 2.73 & & 142 & \\
\hline 173 & 0.13 & & & 34.55 & 192 & \\
\hline 174 & 0.24 & & 3.87 & & 176 & \\
\hline 175 & 0.18 & & & 17.59 & 138 & \\
\hline 176 & 0.18 & & 2.47 & & 173 & \\
\hline 177 & 0.31 & & & 16.82 & 82 & \\
\hline 178 & 0.27 & & 4.38 & & & 239 \\
\hline
\end{tabular}

Notes: Reference range: D-dimer: 0-0.55 mg/L FEU; CEA: 0-5 ng/mL; LDH: II5-220 U/L.

Abbreviations: CEA, carcinoembryonic antigen; $C R C$, colorectal cancer; $\mathrm{LDH}$, lactate dehydrogenase.

\section{Publish your work in this journal}

Cancer Management and Research is an international, peer-reviewed open access journal focusing on cancer research and the optimal use of preventative and integrated treatment interventions to achieve improved outcomes, enhanced survival and quality of life for the cancer patient. The manuscript management system is completely online and includes

\section{Dovepress}

a very quick and fair peer-review system, which is all easy to use. Visit http://www.dovepress.com/testimonials.php to read real quotes from published authors. 